\title{
What Makes Path of Motion Salient?*
}

\author{
STÉPHANIE POURCEL \\ University of Durham
}

\section{Introduction}

The present research develops previous empirical work on the relationship between language and cognition, i.e. linguistic relativity. The approach taken adopts a combination of domain- and structure-centred epistemologies (Lucy 1997), departing from the experiential domain of human motion with a special focus on the dimensions of Path and Manner, and from the lexicalisation patterns available in the French and the English languages to express motion events.

This research expands on French and English linguistic and cognitive data obtained from categorization and elicitation tasks on motion (Pourcel in press a, b). The elicited data indicates that English lexicalises Manner and Path in $85 \%$ of motion verb phrases, whereas French lexicalises Path only in $65 \%$ of verb phrases - leaving Manner optionally lexicalised. This divergence led to the hypothesizing of a weaker level of cognitive salience for Manner amongst French speakers. Categorization tasks on motion with English and French subjects failed to support the hypothesis, as $60 \%$ of responses indicated Path salience in both groups.

This paper reviews these findings and their implications, and presents new experimental findings on memory, attention, inference, and overall cognitive salience of motion dimensions. Experiments include free recall, cue memorisation, and dimensional drawing. Preliminary findings confirm the cognitive prevalence of Path in motion conceptualization - regardless of the native input. Such findings would support the core schematicity of Path in language and in cognition proposed by Talmy (1991).

This paper further addresses the potential reasons behind the centrality of Path in human motion conceptualization. It suggests that, alongside previous proposals such as the cognitive simplicity of Path as shown through earlier acquisition and over-extensions by children (e.g. Choi and Bowerman 1991), other factors are responsible for Path salience. Indeed, single factors alone fail to explain findings

\footnotetext{
* This research has received approval from the Ethics Committee of the University of Durham (UK), and is funded by the Economic \& Social Research Council (UK), award R42200154377.
} 
in other studies reporting cross-linguistic Manner salience approximating 60\% (e.g. Zlatev and David 2003).

The ultimate proposal is that Path receives greater salience depending on a range of variables, e.g. Path telicity, Manner force dynamics, Figure animacy and agency. The current tests aim to monitor these variables and assess their relevance in the differential cognitive salience of motion dimensions in conceptualization.

Empirical efforts attempting to prove the influence of the linguistic encoding of motion dimensions on motion conceptualization may therefore be valid only once these parameters of salience variability have been considered and their effects understood in experimental settings.

\section{Expressing motion events in language}

Non-linguistically speaking, motion events are characterised by a few fundamental features including a Figure (i.e. the moving entity), a Ground (i.e. the spatial reference), a Path (i.e. the directionality followed by the Figure), a Manner (i.e. the fashion in which the Figure moves), a Polarity (i.e. the spatial sequence of the Path), a Cause (i.e. the motivation behind motion), and a Result (i.e. the finality of the motion) - among others.

Linguistically speaking, Talmy's (1985) dual typology for motion expression is particularly relevant to a morphosyntactic appreciation of motion lexicalisation in French and English. It proposes that English - a 'satellite-framed' language typically lexicalises the dimension of Manner in the main verb of the sentence, and the dimension of Path in a satellite element, e.g. a verb particle, so that both Manner and Path are part of the typical English motion verb complex, or verb phrase (henceforth VP), e.g.

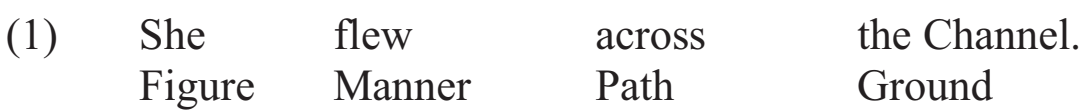

On the other hand, French - a 'verb-framed' language - typically lexicalises the Path dimension in the main verb of the sentence, and the Manner dimension in an optional constituent, so that only Path is part of the typical French motion VP:

$\begin{array}{llll}\text { Elle } & \text { a traversé } & \text { la Manche } & \text { en avion. } \\ \text { Figure } & \text { Path } & \text { Ground } & \text { Manner } \\ \text { She } & \text { crossed } & \text { the Channel } & \text { by plane. }\end{array}$

The validity of Talmy's binary typology, as exemplified in (1) and (2), was confirmed in controlled elicitation tasks in $85 \%$ of instances in English $\left(\mathrm{N}_{\text {[motion }}\right.$ sentences] $=1382)$ and in $65 \%$ of instances in French $\left(\mathrm{N}_{\text {[motion sentences] }}=1800\right) .{ }^{1}$ Albeit a matter of degree, these findings indicate a clear cross-linguistic difference of a structural nature. This structural state of affairs suggests

\footnotetext{
${ }^{1}$ Written elicitation tasks used 45 motion scenes shown on a TV set $\left(\mathrm{N}_{\text {English }}=31, \mathrm{~N}_{\text {French }}=40\right)$.
} 
differential linguistic foregrounding of Manner in French and English. Indeed, the French language tends to background the element of Manner to the extent that it is often left unsaid altogether - unlike in (2), e.g.

$\begin{array}{lll}\text { L'oiseau } & \text { est sorti } & \text { de sa cage. } \\ \text { Figure } & \text { Path } & \text { Ground } \\ \text { The bird } & \text { exited } & \text { its cage. }\end{array}$

Encoding Manner may render sentences heavy, clumsy, and even redundant, so that sentences like (2) may be judged ungrammatical, e.g. ${ }^{2}$

\begin{tabular}{|c|c|c|c|c|}
\hline$? 28 \%$ & $\begin{array}{l}\text { Les enfants } \\
\text { Figure } \\
\text { The children }\end{array}$ & $\begin{array}{l}\text { vont } \\
\text { Path } \\
\text { go }\end{array}$ & $\begin{array}{l}\text { à l'école } \\
\text { Ground } \\
\text { to school }\end{array}$ & $\begin{array}{l}\text { en trépignant. } \\
\text { Manner } \\
\text { stamping their feet. }\end{array}$ \\
\hline $237.5 \%$ & $\begin{array}{l}\text { Titi } \\
\text { Figure } \\
\text { Tweety }\end{array}$ & $\begin{array}{l}\text { sort } \\
\text { Path } \\
\text { exits }\end{array}$ & $\begin{array}{l}\text { de sa cage } \\
\text { Ground } \\
\text { its cage }\end{array}$ & $\begin{array}{l}\text { en volant. } \\
\text { Manner } \\
\text { flying. }\end{array}$ \\
\hline
\end{tabular}

The reluctant codability of Manner in French is further mirrored at the lexical level, as verb-framed languages are indeed poorly equipped with lexical referents to Manner, as compared to satellite-framed languages, both quantitatively and qualitatively (Slobin 1997). The French construal of motion events highlights instead the static relations existing between scene components, e.g. Ground details and Figure states (e.g. psychological, locational, etc.).

Overall, both language types elaborate different discursive styles whereby different dimensions of motion construals receive foregrounding in linguistic expression. Linguistically speaking, the Path dimension is highly codable in both language types - though English may more readily accumulate Path and Ground combinations than French. On the other hand, Manner is highly codable in English only, and is represented by the lexicalisation pattern for motion expressions, whereas Manner is less codable in French and is typically left unsaid in natural discourse unless relevant to the arguments.

\section{Dimensional categorization of motion in cognition}

The question under debate, then, is whether speakers of different languages conceptualize motion differently because of the construals elaborated by their native language. Prior to investigating this question and the extent of its potential, it is fundamental to establish an understanding of motion conceptualization in general cognitive terms - one that is same for all speakers, regardless of their native tongue.

\footnotetext{
${ }^{2}$ Grammaticality judgements were provided by 64 native French speakers, in collaboration with Anetta Kopecka, Laboratoire Dynamique du Langage, Université de Lyon 2, France.
} 


\subsection{Methodology}

Pourcel (in press a, in press b) has reported cognitive data testing English and French native speakers on cognitive visualisation tasks $\left(\mathrm{N}_{\text {English }}=64, \mathrm{~N}_{\text {French }}=75\right)$. Categorization experiments were implemented, requiring native subjects to judge mute visual stimuli (in the form of video clips) in terms of similarity, e.g.

(6) a. a man running up a hill

b. a man running down a hill

c. a man walking up a hill

The test comprised fifteen such triads, with differing Manner and Path types (but invariant Grounds and Figures), always organised so that two distinct pairs would correspond to Path similarity (e.g. 3a and $3 \mathrm{c}$ above) and to Manner similarity (e.g. $3 \mathrm{a}$ and $3 \mathrm{~b}$ ). Path types included instances of up, down, into, out of, across, along directions, and Manner types included instances of walking, running, tiptoeing, limping, cycling, climbing, kicking, pushing, pulling, and more.

\subsection{Experimental results}

This experiment revealed striking similarities in cognitive performance, with both language groups favouring Path associations in 55.5\% of choices in the English sample, and in $53.5 \%$ in the French sample - against $40.5 \%$ of English Manner choices, and $39.5 \%$ of French choices. ${ }^{3}$ These patterns suggest that Path may be slightly more cognitively salient than Manner in human motion conceptualization.

A closer examination further revealed consistent response ranking across triadic stimuli type (see Graph 1), so that some triads yielded over $80 \%$ of Manner responses, whereas others obtained under $20 \%$ of Manner responses. This suggests that some intrinsic characteristics of the motion scenes trigger differential cognitive salience for Path and Manner. In other words, Path and Manner receive different levels of salience in conceptualization depending on the nature of the motion scenes themselves. These salience factors - which are not linguistic must be identified and understood prior to investigating language as an additional factor influencing motion conceptualization. In the present case, the factors identified are conceptual and correspond to Path telos and to Manner force dynamics.

\footnotetext{
${ }^{3}$ Statistics do not equate to $100 \%$ in either language group, as some choices correspond to 'impossible' associations. The test - by its monitoring nature - did not present a target item with two alternate items.
} 
Graph 1. Ranked Manner responses.

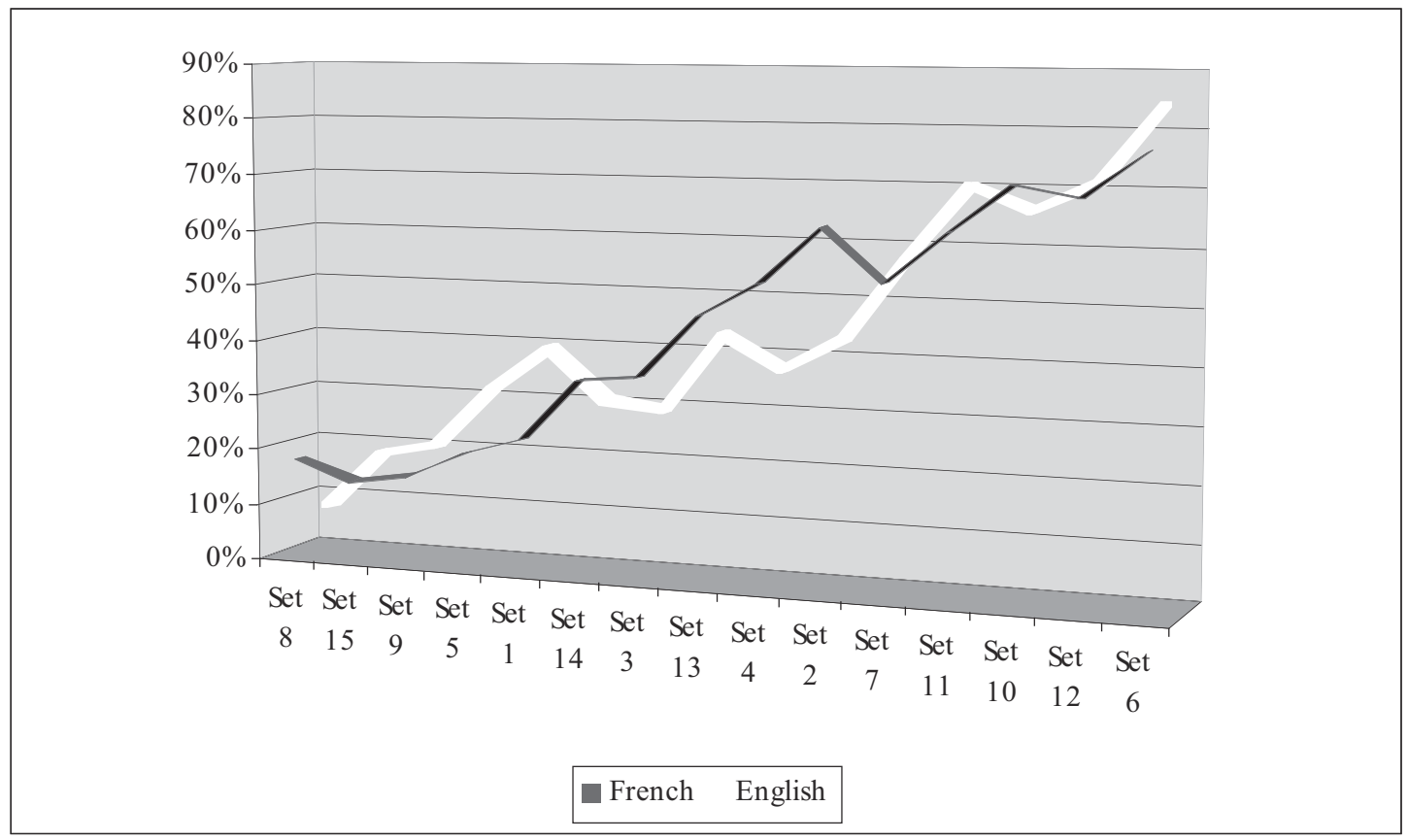

\subsection{Path telos}

As highlighted by Aske (1989), there exists two distinct types of Path, namely telic and atelic (or locative) Paths. Telicity entails an end-point or the crossing of a boundary, so that (7) represents a telic type of Path, and (8) an atelic one:

(7) We walked into the room.

(8) We walked along the beach.

Both language groups displayed a clear correlation between Path type and association type, whereby telic stimuli trigger Path preferences and atelic stimuli trigger Manner preferences (see Table 1).

Table 1. Proportion of association types relative to telicity.

\begin{tabular}{lcccc}
\hline & \multicolumn{2}{c}{ Telic sets } & \multicolumn{2}{c}{ Atelic sets } \\
\hline & Path & Manner & Path & Manner \\
\hline English $(\mathrm{N}=64)$ & $64 \%$ & $36 \%$ & $45 \%$ & $54 \%$ \\
French $(\mathrm{N}=75)$ & $64 \%$ & $36 \%$ & $43 \%$ & $56 \%$ \\
\hline
\end{tabular}

We may infer from these figures that there is a definite link between the conceptual salience of either Manner or Path and telicity in motion. It is possible to offer a preliminary explanation for this state of affairs through an understanding of directionality. The notion of directionality in real-life, human motion combines with the notion of agent intentionality, in the sense that human behaviour is goal- 


\section{Stéphanie Pourcel}

driven. Directionality in human motion may be understood to represent the very goal of motion - at least in typical cases. Such a suggestion would entail that the Path dimension overrides the Manner dimension in the cognitive appreciation of human motion as a general rule. The purposeful attitude on behalf of the agent presupposed by directionality is moreover somewhat lacking in the dimension of Manner - except possibly in ad hoc Manner types (e.g. tiptoeing bare-feet on gravel to avoid pain or jumping to get something out reach) - even when Path is partly inherent and invoked by other features such as Ground.

With humans being meaning-seeking creatures, it appears very likely that the purpose-loaded dimension of motion should therefore be the dimension receiving higher levels of cognitive salience across species members and hence across language groups. As a rule, the end justifies the means, and it is possible that the means, or the Manner of motion in this case, is secondary in human actions. Furthermore, in the case of a decontextualised task, such as the present categorization tests, it is also possible that subjects found an even greater need to reconstruct, or simply to identify or infer, agent intentionality in order to make sense out of the stimuli, and out of the task overall.

To explain the data in Table 1, it is evident that in telic cases, the agent's directional purpose is rendered particularly explicit, and hence salient. On the other hand, when directionality is unclear and intentionality is hence uninferable, subjects' performance granted Manner higher salience.

It is possible that Manner is never quite the most cognitively salient element in motion. Rather, Path may always be relatively more salient, unless it is atelic and the agent intentionality is unclear. Further, this suggestion may be valid to the limited extent that the motion agent is human, or at least animate, on two grounds, (a) intentionality is a cognitive ability requiring a cerebral creature, and (b) the natural human tendency to self-project entails that empathy on an intentional level is possible so long as the self-projection recipient conforms to the original, i.e. it has to be animate, and human ideally. This possibility would predict that similar experiments on non-intentioned moving agents (e.g. inanimate agents) would fail to reproduce the Path salience reported in the present research. This is indeed the case in studies implemented with the Max Planck Institute of Psycholinguistics's elicitation tool using 2-D digital vegetables in motion scenes. These studies report an overall two third preference for Manner, the opposite of the present findings (e.g. Zlatev and David 2003).

\subsection{Manner force dynamics}

The human body can perform motion in a number of different ways, or types of Manner. Manner types differ from one another depending on various aspects, such as the body part(s) used, instruments or vehicles, force dynamics, inherent directionality, the presence of an axis, actual displacement, and so on.

Based on the stimuli used in the present experimental set-up, I suggest a broad classification of Manner types with 3 categories of force features: (a) default, (b) forced, and (c) instrumental Manner types. Default Manner types refer to the 
expected Manner for performing a motion, e.g. walking (humans), running (humans), crawling (babies), flying (birds), rolling (balls), e.g.

(9) He walked into the house.

Forced Manner types involve some conscious and intentional effort, or some form of physical impediment, so that the Manner of motion involves a level of difficulty in performance, e.g. hopping, skipping, kicking, throwing, limping, bouncing, marching, zigzagging, waltzing, e.g.

(10) She tiptoed up the stairs.

Finally, instrumental Manner types involve an extra element besides the human body used to perform the motion, e.g. cycling, rowing, ballooning, skating, e.g.

(11) We skied down the slope.

The data from this small-scale study already suggest a consistent correlation between neutrality of Manner (i.e. default) and low Manner scores on the one hand, and force and instrumentality features and higher Manner scores on the other hand (see Table 2).

Table 2. Proportions of association types relative to force features.

\begin{tabular}{lcccccc}
\hline & \multicolumn{2}{c}{ Default } & \multicolumn{2}{c}{ Forced } & \multicolumn{2}{c}{ Instrumental } \\
\hline & Path & Manner & Path & Manner & Path & Manner \\
\hline English $(\mathrm{N}=64)$ & $76 \%$ & $24 \%$ & $51 \%$ & $49 \%$ & $41 \%$ & $59 \%$ \\
French $(\mathrm{N}=75)$ & $74 \%$ & $26 \%$ & $50 \%$ & $50 \%$ & $48 \%$ & $52 \%$ \\
\hline
\end{tabular}

These figures indicate that Path of motion receives higher cognitive salience than Manner when Manner types correspond to default expectations for motion performance, whereas attention is more strongly focused on Manner itself when force features involve efforts and/ or instruments.

This confirms the above suggestion that Path is more centrally salient than Manner in motion conceptualization, as Manner salience only obtains when default expectations are violated. However, Manner is never overwhelmingly more salient than Path, and the differences in forced and instrumental scores for Path and Manner choices do not yield statistical significance.

\subsection{Summary}

The identification of the above features as factors of influence on dimensional salience in motion conceptualization enables cognitive predictions for behaviour combining both Path telos and Manner force dynamics (see Graph 2). Indeed, we may predict the following: 
(12) $[$ +telicity $][$-force $]=$ higher Path salience

(13) $\quad$-telicity $[$-force $]=$ higher Path salience but lower than in case (12)

(14) $[+$ telicity $][+$ force $]=$ mixed Path and Manner salience

(15) $\quad[$-telicity $][+$ force $]=$ higher Manner salience

Graph 2. Proportions of Path associations relative to force features and telicity ${ }^{4}$

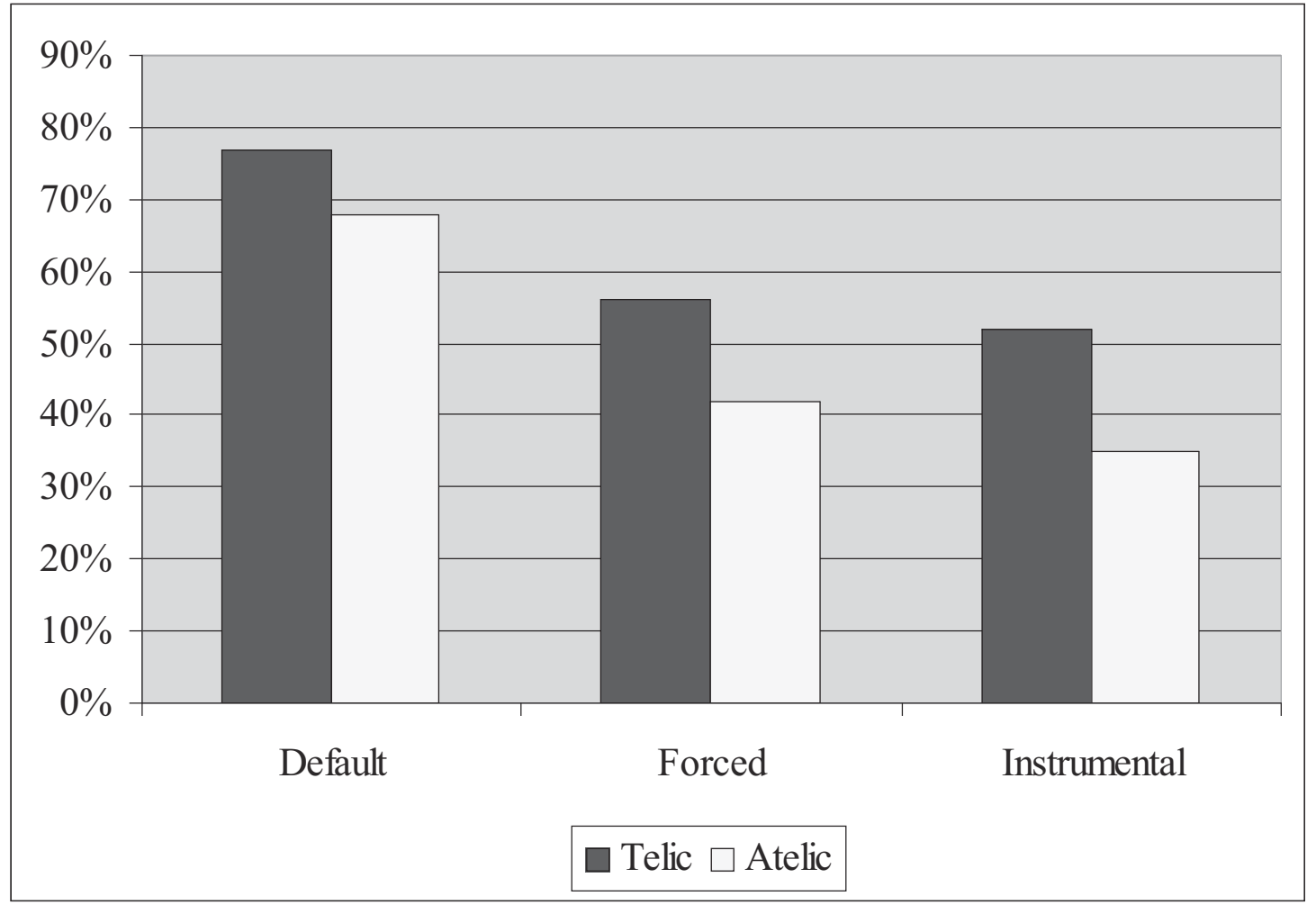

It therefore appears, at preliminary glance, that what makes Path cognitively salient in motion conceptualization are Path telicity, default Manner types, human Figures, and agent intentionality.

\section{Linguistic relativity}

Based on the above understanding, further experiments were implemented testing memory and conceptualization through drawing. These experiments are at a pilot stage and the results are only suggestive therefore. Nonetheless, methodologically speaking, these tests offer new ways of investigating language effects on cognition. Preliminary results do suggest differences between English and French speakers following the patterns of their native languages.

\footnotetext{
${ }^{4}$ This graph illustrates the results of both language groups conflated together.
} 


\subsection{Conceptualising motion through drawing}

\subsubsection{Methodology}

This experiment asked subjects $\left(\mathrm{N}_{\text {English }}=18, \mathrm{~N}_{\text {French }}=8\right)$ to produce drawings of 5 short video clips displaying motion scenes a few seconds long, as follows:

(16) A man running down a flight of stairs.

(17) A man jogging along a street and into a house.

(18) A man limping towards a woman sat down on a sofa.

(19) A man kicking a door shut.

(20) A man diving into a swimming pool.

In drawing tasks, subjects try to render the stimuli elements that they perceive to be salient so that drawings represent fair translations of the stimuli. With motion, subjects have to render an unbounded, dynamic 3-D image onto a bounded, static 2-D format; this means that subjects must select in and out features relevant to their conceptualization of the stimuli. For instance, Manner of motion may be particularly difficult to draw, e.g. limping, so that an attempt to render limping would entail that Manner has been selected as a particularly salient feature of the stimulus. Likewise, Path entails some change of location, and is therefore a dynamic concept that is not drawable in the sense that static entities, such as Grounds, are. Therefore, subjects would have to add arrows or dotted lines - not seen in the stimulus - were they to find Path a salient feature in their conceptualization of the stimulus.

\subsubsection{Results}

Few differences were observed across language groups in the drawing of Grounds, Figures, and Manners. Differences appeared in the rendering of Path, which French subjects drew more systematically, using lines, arrows, and segmented Figures. Likewise, French subjects more readily provided details of a contextual nature, i.e. descriptions of background settings (e.g. vegetation, sunshine). Finally, this task also tested the predictions in (12)-(15). The Manner types displayed in the video stimuli displayed a gradation in force features:

$$
\begin{aligned}
& \mathrm{RUN} \rightarrow \mathrm{JOG} \rightarrow \mathrm{LIMP} \rightarrow \mathrm{DIVE} \rightarrow \mathrm{KICK} \\
& {[- \text { force }] \rightarrow \rightarrow \rightarrow \rightarrow \rightarrow \rightarrow[+ \text { force }]}
\end{aligned}
$$

Ironically, drawing default Manner types should be somewhat easier, which may encourage results going against the predictions. However, the reverse obtained so that subjects' performances strongly confirmed the force-based predictions for Manner salience in conceptualization (see Table 3). 
Table 3. Proportions of Manner types drawn

\begin{tabular}{lccccc}
\hline & Default & & & & Forced \\
\hline & Run & Jog & Limp & Dive & Kick \\
\hline English $(\mathrm{N}=18)$ & $50 \%$ & $56 \%$ & $67 \%$ & $72 \%$ & $100 \%$ \\
French $(\mathrm{N}=8)$ & $25 \%$ & $63 \%$ & $75 \%$ & $88 \%$ & $100 \%$ \\
\hline
\end{tabular}

With respects to Path, (12)-(15) would predict telic Path types to be drawn more consistently. However, all video clips displayed telic motion events. Yet, a gradation in degree of telicity is notable, with Path types showing a progression, e.g. along, less consistently drawn than punctual or sudden Path types, with an obvious change of location or end point, e.g. into. In other words, the more telic the Path, the more salient it seems to be in cognition (see Table 4).

Table 4. Proportions of Path types drawn

\begin{tabular}{lccccc}
\hline & {$[-$ telos $]$} & & & & {$[+$ telos $]$} \\
\hline & Down & Along & Shut & Towards & Into \\
\hline English $(\mathrm{N}=18)$ & $39 \%$ & $44 \%$ & $50 \%$ & $56 \%$ & $67 \%$ \\
French $(\mathrm{N}=8)$ & $25 \%$ & $63 \%$ & $63 \%$ & $75 \%$ & $75 \%$ \\
\hline
\end{tabular}

\subsection{Memorizing motion dimensions}

\subsubsection{Methodology}

The memory tests attempted to contextualize motion scenes in real-life settings, using a 4 $\frac{1}{2}$ minute extract from Charlie Chaplin's City Lights - comprising numerous motion events with various types of Manner and Path. Subjects $\left(\mathrm{N}_{\text {English }}\right.$ $=14, \mathrm{~N}_{\text {French }}=8$ ) performed free prose and recognition recall tasks.

\subsubsection{Results}

Error rates in accurate memorizing in the free prose recall are low, overall, yet they reveal different patterns across the language groups (see Table 5). Languagebased predictions would entail that motion (and Manner) may be more closely attended to by native English speakers - given the high codability of its dimensions in English - whereas Figure states, Grounds, and overall context may be more readily foregrounded in French cognition as a result of their linguistic foregrounding in French lexicalisation patterns for motion encoding.

Table 5. Proportions of errors in the free prose recall. ${ }^{5}$

\begin{tabular}{lccc}
\hline & Agent motion & Object motion & Agent state \\
\hline English $(\mathrm{N}=14)$ & $7 \%$ & $9 \%$ & $11 \%$ \\
French $(\mathrm{N}=8)$ & $13 \%$ & $17 \%$ & $7 \%$ \\
\hline
\end{tabular}

These predictions are further validated by the recognition results (see Table 6).

\footnotetext{
5 Agentive and object motion were distinguished on the basis of the prior prediction concerning the higher salience of Path in animate motion in human cognition.
} 
Table 6. Proportions of errors in the recognition recall.

\begin{tabular}{lcl}
\hline & Manner & Path \\
\hline English $(\mathrm{N}=14)$ & $15 \%$ & $80 \%$ \\
French $(\mathrm{N}=8)$ & $32 \%$ & $37 \%$ \\
\hline
\end{tabular}

One Manner question was excluded from this count as it skewed the statistics. It asked whether Chaplin runs at a critical point when a character is about to jump in a river to commit suicide. The right answer was positive. The English error rate was an unusual $55 \%$ (vs. $25 \%$ for the French). One comment was additionally often voiced by English subjects: "Chaplin never runs!" This may suggest that English speakers pay so much attention to Manner of motion, that people may be characterised and identified partly by the way they move. Such a characterisation means that some English answers may not have been actual recall, but inference, in which case their general characterisation of Chaplin's default Manner of motion induced them into error when an unexpected Manner type occured. The fact that French speakers did not make half as many errors is similarly suggestive that partial attention only to Manner did not generate this error to the same extent.

Finally, the figures reported on Path errors beg further comment. This rate corresponds to one question only, which asked whether a character took his shoes off before rescuing Chaplin from drowning in the river. The answer was negative. Examining the motion scene more closely, the act of taking one's shoes off is easily inferred from a preceding set of Manners of motion, e.g. sitting down, crouching, bending over, reaching for one's shoes. In the film, the character had just taken off his jacket, and then decided to sit on a bench, where he crossed his legs, and reached for one shoe - though he never undid either shoe in the end. Again, it may be suggested from the substantial difference in error rates, namely $43 \%$, that English speakers inferred the result of having ones shoes off from the Manners of motion that preceded that result. The explicitness of those Manners led subjects to the false deduction that the shoes had come off. One possibility for this false inference by English speakers may relate to a higher level of attention paid to Manner by English native speakers. It is also worth noting that this particular error was also made in the free prose recall to the extent that some English subjects speculated that the character put his shoes back on at the end of the film. On the other hand, French subjects never made such a suggestion.

\section{Conclusion}

Through experimental set-ups, the present research has identified intrinsic motion properties responsible for the higher salience of Path in human motion conceptualization - telicity, default Manner types, agent animacy and intentionality. These properties were consistent findings in the categorization and drawing tasks. The memory tests aimed at testing linguistic relativity, asking whether Manner is differentially attended to across the 2 language groups. It provided an integration of motion scenes within a human context, and yielded differences concordant with the hypothesis that Manner would be more salient in English speakers' cognition. 
This finding was reinforced by unexpected errors made by the English group on unexpected Manner types and Path inferences.

Importantly, these tests are only at a pilot stage and used small samples of native speakers. As such, they suggest new experimental approaches to the study of linguistic relativity and motion conceptualization. Nonetheless, the results are already suggestive, and further testing may reveal more consistent differences, and confirm some level of relativism in the domain of motion between French and English native speakers.

\section{References}

Aske, Jon. 1989. Path predicates in English and Spanish: A closer look. Proceedings of the 15th Annual Meeting of the Berkeley Linguistics Society, 1-14. Berkeley, CA: Berkeley Linguistics Society.

Choi, Soonja and Melissa Bowerman. 1991. Learning to express motion events in English and Korean: The influence of language-specific lexicalisation patterns. Cognition 41: 83-121.

Lucy, John. 1997. Linguistic relativity. Annual Review of Anthropology 26: 291312.

Pourcel, Stéphanie. in press a. Rethinking 'thinking for speaking'. Proceedings of the 29th Annual Meeting of the Berkeley Linguistics Society. Berkeley, CA: Berkeley Linguistics Society.

Pourcel, Stéphanie. in press b. Motion in language and cognition. In A. Soares da Silva (ed.) Linguagem, Cultura e Cognição: Estudos de Linguistica Cognitiva. Coimbra: Almedina.Slobin, Dan. 1997. Mind, code, and text. In J. Bybee, J. Haiman and S.A. Thompson (eds.) Essays on Language Function and Language Type: Dedicated to T. Givón, 437-67. Amsterdam: John Benjamins.

Talmy, Leonard. 1985. Lexicalisation patterns: Semantic structure in lexical forms. In T. Shopen (ed.) Language Typology and Syntactic Description, vol. 3, 57-149. Cambridge: Cambridge University Press.

Talmy, Leonard. 1991. Path to realisation: A typology of event conflation. Proceedings of the 17th Annual Meeting of the Berkeley Linguistics Society, 480-520. Berkeley, CA: Berkeley Linguistics Society.

Zlatev, Jordan and Caroline David. 2003. Manner vs. Path in the language and cognition of Swedish and French speakers. Conference presentation, Language, Culture \& Cognition, Braga, Portugal, July 18, 2003.

Stéphanie Pourcel

School of Linguistics and Language

University of Durham

Durham, DH1 3JT

United Kingdom

s.s.pourcel@durham.ac.uk 